\title{
Salmonella Newport Bacteremia in a 12-Day-Old Infant
}

\author{
Abhijit Singh, MBBS, Thad Wilkins, MD, and Robert R. Schade, MD
}

In the United States, Salmonella infections (salmonellosis) cause multiple medical problems. Although the most common presenting symptom is diarrhea, bacteremia can also occur. An estimated 1.4 million cases of salmonellosis occur annually in the United States. We present a case of Salmonella bacteremia in a 12-day old infant. We discuss the presenting signs, symptoms, and management strategies for a patient younger than 28 days old (neonate) presenting with fever and diarrhea. (J Am Board Fam Med 2011;24:214-217.)

Keywords: Bacteremia, Case Report, Family Medicine, Newborn, Salmonellosis

In the United States, Salmonella infections (salmonellosis) cause multiple medical problems. Although the most common presenting symptom is diarrhea, bacteremia can also occur. An estimated 1.4 million cases of salmonellosis occur annually in the United States, with infections most commonly caused by the Typhimurium and Enteritidis salmonella species. ${ }^{1}$ The third most common salmonella serotype in the United States is Salmonella newport. ${ }^{1}$ The number of confirmed S. newport infections reported by the Centers for Disease Control and Prevention increased from 1,584 of 34,608 (5\%) to 3,152 of 31,607 (10\%) of all Salmonella infections during the period of 1997 to $2002 .{ }^{1}$ We present a case of $S$. newport causing bacteremia in a 12 -dayold infant. To our knowledge there have been to date no published case reports of $S$. newport in neonates in the United States. We discuss the presenting signs, symptoms, and management strategies for a patient younger than 28 days old (neonate) presenting with fever and diarrhea.

This article was externally peer reviewed.

Submitted 29 June 2010; revised 11 October 2010; accepted 22 October 2010.

From the Department of Family Medicine, Medical College of Georgia, Augusta.

Funding: none.

Conflict of interest: none declared.

Corresponding author: Thad Wilkins, MD, Associate Professor, Department of Family Medicine, Medical College of Georgia, 1120 Fifteenth Street, HB-4032, Augusta, GA 30912 (E-mail: twilkins@mcg.edu).

\section{Case Report}

A 12-day-old infant was brought to the emergency department at a community hospital by his mother after he had 10 loose, yellowish-green stools and a documented fever of $101^{\circ} \mathrm{F}\left(38.3^{\circ} \mathrm{C}\right)$ by rectal thermometer. According to his mother, there was no history of any blood or mucus in the stool and there was no vomiting. The infant lived with his mother, father, uncle, and 1-year-old cousin. His cousin was recently diagnosed with an uncomplicated Salmonella diarrheal disease (the salmonella species causing the disease was unknown), and he was being treated empirically with oral antibiotics. The infant's mother smoked cigarettes but not inside the house. The family had no pets or livestock at home. The prenatal course was uncomplicated and he was born full term (39 weeks estimated gestational age) through an uncomplicated, normal, spontaneous vaginal delivery. His birth weight was $3.9 \mathrm{~kg}$. He received his first hepatitis $B$ vaccination and vitamin $\mathrm{K}$ at birth, and an uncomplicated circumcision was performed before discharge. His mother started him on bottle feedings with Enfamil (Mead Johnson \& Co, LLC, Evansville, IN) in the nursery and she continued this after discharge from the hospital.

In the emergency department, the infant's weight was $3.8 \mathrm{~kg}$ (a $2.6 \%$ decrease from his birth weight). During the examination he was crying but was easily consolable by his mother. Anterior fontanel was soft and flat. Mucus membranes were moist; extremities were warm and well-perfused 
(capillary refill took $<2$ seconds). No neck stiffness was observed, and he had an umbilical stump with no signs of infection such as erythema or exudates. His neurological, cardiovascular, and respiratory examinations were normal.

Blood, urine, and stool specimens were obtained for analysis and culture. Complete blood count showed a white blood cell count of 15.7 thousand/ $\mathrm{mm}^{3}$ (normal, $4.5-11.0$ thousand $/ \mathrm{mm}^{3}$ ), with a differential of $60 \%$ neutrophils and $20 \%$ lymphocytes. Hemoglobin level was 16.4 g/dL (normal, 14.5$24.0 \mathrm{~g} / \mathrm{dL}$ ). Urinalysis was normal. A lumbar puncture was performed in the emergency department, and cerebral spinal fluid (CSF) was sent for analysis and culture. The CSF showed a glucose level of 68 $\mathrm{mg} / \mathrm{dL}$ (normal, $40-70 \mathrm{mg} / \mathrm{dL}$ ) and a protein level of $88 \mathrm{mg} / \mathrm{dL}$ (normal, $20-170 \mathrm{mg} / \mathrm{dL}$ ); one red blood cell and 4 nucleated cells were noted. Chest radiograph was normal. He was euvolemic and did not receive intravenous fluids because he was tolerating feedings by mouth. After intravenous administration of $200 \mathrm{mg}$ of ampicillin $(50 \mathrm{mg} / \mathrm{kg})$ and $9 \mathrm{mg}$ of gentamicin $(2.5 \mathrm{mg} / \mathrm{kg})$, he was transported via ambulance to our tertiary referral center for further evaluation and management.

Upon arrival at our facility and 8 hours after his initial dose of antibiotics, he received intravenously $380 \mathrm{mg}$ of ampicillin $(100 \mathrm{mg} / \mathrm{kg})$ every 8 hours and $16 \mathrm{mg}$ of gentamicin $(4 \mathrm{mg} / \mathrm{kg})$ daily. The patient tolerated formula (Good Start, Gerber/Nestlé, Fremont, MI) and there were no signs or symptoms of volume contraction; therefore, he did not receive intravenous fluids during his hospitalization. By hospital day 3 the diarrhea and fever had resolved. On hospital day 4 the initial blood and stool cultures obtained from the community hospital were reported as positive for $S$. Newport. The following day, antibiotic sensitivity and susceptibilities showed the organism was sensitive to ampicillin and cefotaxime. Ampicillin and gentamicin were discontinued and he was treated intravenously with cefotaxime 250 $\mathrm{mg}(50 \mathrm{mg} / \mathrm{kg})$ for the next 10 days. He was discharged after 14 days (total) of intravenous antibiotics. At his outpatient appointment, 3 days after discharge, he was doing well without fever, diarrhea, or vomiting, and he was tolerating oral formula. His weight was $4.5 \mathrm{~kg}$ (a $15 \%$ increase from birth weight).

\section{Discussion}

Fever in an infant younger than 90 days old is defined as a temperature $>38^{\circ} \mathrm{C}$ or $100.4^{\circ} \mathrm{F}$; and rectal measurement is the gold standard. ${ }^{2}$ Approximately $20 \%$ of fevers in young children have no apparent source, and fever is the third most common reason for emergency department visits among children in the United States. ${ }^{3,4}$ Infection is the most common cause of fever in infants, with bacterial and viral pathogens causing most infections. ${ }^{5}$ Common bacterial infections include group B streptococcus, Escherichia coli, Haemophilus influenza type B, Neisseria meningitidis, Listeria monocytogenes, Salmonella, Staphylococcus aureus, and Streptococcus pneumoniae. ${ }^{5,6}$ Most viral infections are selflimited and are not associated with significant morbidity or mortality. Common viral pathogens that cause neonatal fever include enterovirus, herpes simplex virus, influenza, respiratory syncytial virus, and rotavirus. ${ }^{5,6}$

Evaluation of an infant with fever should include a complete history and physical examination. ${ }^{6}$ The physician should ask how the temperature was taken because the accuracy of the temperature depends on the method of measurement. The physician should ask about recent medication use, including antipyretics and prior antibiotics. The history should include information about immunization status, medical history, or prematurity. Social history should include any exposure to sickness and travel history. The review of systems includes questions regarding sweating, decreased appetite, lethargy, irritability, and seizures. Physical examination should rule out toxic appearance, eg, pale skin, cyanosis, lethargy, inconsolability, tachypnea, and poor capillary refill. ${ }^{7}$ Other concerning examination findings are reduced skin turgor, bulging or sunken fontanelle, neck stiffness or rigidity, dry mucous membranes, nasal flaring, or focal neurologic signs. ${ }^{7}$

Blood culture, urinalysis, and urine culture are recommended for all febrile infants younger than 90 days old. In addition, a white blood cell count with differential and CSF cell counts and culture should be considered for infants younger than 60 days old. ${ }^{6}$ Additional testing is recommended as clinically indicated, eg, chest radiograph if there are respiratory signs or stool culture if diarrhea is present. ${ }^{6}$ Intravenous antibiotics are recommended for infants younger than 28 days old or for infants who have a toxic appearance, high risk for serious bacterial infection, abnormal complete blood cell count or urinalysis test results, or uncertain or unreliable follow-up. ${ }^{6}$ A typical empiric regimen is 
ampicillin $(50 \mathrm{mg} / \mathrm{kg}$ intravenously or $100 \mathrm{mg} / \mathrm{kg}$ intravenously for suspected meningitis) plus either a third-generation cephalosporin, eg, cefotaxime $(50 \mathrm{mg} / \mathrm{kg}$ intravenously or 75 to $100 \mathrm{mg} / \mathrm{kg}$ intravenously for suspected meningitis) or gentamicin ( $2.5 \mathrm{mg} / \mathrm{kg}$ intravenously). Ceftriaxone is not recommended for use in infants younger than 28 days old secondary to the risk of unconjugated hyperbilirubinemia. ${ }^{6}$

S. newport is a gram-negative, facultative, intracellular rod that infects cattle and is often transmitted by fecal-oral contamination or consuming unpasteurized milk or undercooked meat or eggs. ${ }^{8}$ Several case reports have indicated severe neonatal infections with $S$. newport in African countries such as Kenya, Morocco, and Tanzania. ${ }^{9}$ This case report is unique because, in our review of the literature, we did not find any published case reports of $S$. Newport in neonates in the United States. The Centers for Disease Control and Prevention has reported antibiotic resistance of $S$. newport in the United States because of the development of multidrug resistance from the use of antibiotics given to cattle. ${ }^{10}$ Reservoirs for nontyphoidal Salmonella serotypes are poultry; livestock; reptiles (turtles, lizards, iguanas, frogs, snakes); and other pets, and the incubation period for Salmonella infection in humans is 12 to 36 hours for gastroenteritis and 7 to 14 days for enteric fever.

Management of salmonellosis with antibiotics is not indicated in cases of noninvasive gastroenteritis because this can prolong colonization. ${ }^{11}$ Treatment for salmonella bacteremia is recommended in infants younger than 3 months of age or patients who have chronic gastrointestinal diseases, malignancies, hemoglobinopathies, and/or HIV infection. Broad-spectrum intravenous antibiotics, such as third-generation cephalosporins, should be initiated in patients who have osteomyelitis, abscess, meningitis, and bacteremia. ${ }^{12}$ Specific antibiotic susceptibility will be determined by culture and sensitivity assays, and the appropriate antibiotic should be administered intravenously for 2 to 4 weeks. ${ }^{11}$ For invasive but nonfocal infections such as bacteremia or enteric fever, treatment with 10 to 14 days of cephalosporin or fluoroquinolones is recommended ${ }^{11}$; however, fluoroquinolones are contraindicated in children because of the risk of complications such as tendon rupture..$^{13}$ Prevention of Salmonella disease is the most effective way to avoid infection and is achieved by washing hands, avoiding close contact with pets such as lizards and turtles, avoiding drinking unpasteurized milk, and avoiding consuming undercooked meats and eggs. 1,14

\section{Conclusion}

Neonatal fever is a common cause of emergency department visits. Appropriate workup of neonatal fever often includes complete blood count, urinalysis, evaluation of CSF, blood culture, urine culture, CSF culture, chest radiograph, and stool cultures as clinically indicated. ${ }^{5}$ Immediate management with intravenous antibiotics should be initiated in cases of suspected sepsis pending culture results. Once culture results are obtained, antibiotics with adequate susceptibilities should be continued for the next 10 to 14 days. ${ }^{5}$

Salmonella is the most common preventable diarrheal illness in the United States, but the prophylactic use of antibiotics in the farming industry has led to development of antibiotic- resistant strains of Salmonella. ${ }^{6,12}$ No antibiotics are recommended for asymptomatic, noninvasive gastroenteritis unless the infant is younger than 90 days of age, has chronic gastrointestinal diseases or hemoglobinopathies, or is immunocompromised. ${ }^{11}$ Noninvasive infections such as bacteremia and enteric fever can be treated with cephalosporins or fluoroquinolones for 14 days, although the use fluoroquinolones should be avoided in children because of the risk of complications. ${ }^{11}$ A 4-week treatment with cephalosporins is recommended for invasive salmonellosis, eg, osteomyelitis or abscess. ${ }^{11}$ Prevention is the best way to avoid Salmonella infections and can be accomplished by frequent hand washing and avoiding consuming undercooked meat and unpasteurized milk. ${ }^{1}$

\section{References}

1. Centers for Disease Control and Prevention. Outbreak of multidrug-resistant Salmonella Newport-United States, January-April 2002. MMWR Morb Mortal Wkly Rep 2002;51:545-8.

2. Bonadio WA, Smith DS, Sabnis S. The clinical characteristics and infectious outcomes of febrile infants aged 8 to 12 weeks. Clin Pediatr 1994;33:95-9.

3. Baraff LJ. Management of infants and young children with fever without source. Pediatr Ann 2008; 37:673-9.

4. Nawar EW, Niska RW, Xu J. National Hospital Ambulatory Medical Care Survey: 2005 emergency department summary. Adv Data 2007;(386):1-32. 
5. Ishimine P. Fever without source in children 0 to 36 months of age. Pediatr Clin North Am 2006;53:16794.

6. Ishimine P. The evolving approach to the young child who has fever and no obvious source. Emerg Med Clin North Am 2007;25:1087-115, vii.

7. Luszczak M. Evaluation and management of infants and young children with fever. Am Fam Physician 2001;64:1219-26.

8. Weir E, Dore K, Currie A. Enhanced surveillance for Salmonella Newport. CMAJ 2004;171:127-8.

9. Brooks JT, Shapiro RL, Kumar L, et al. Epidemiology of sporadic bloody diarrhea in rural Western Kenya. Am J Trop Med Hyg 2003;68:671-7.

10. Karon AE, Archer JR, Sotir MJ, Monson TA, Kazmierczak JJ. Human multidrug-resistant Salmonella
Newport infections. Wisconsin, 2003-2005. Emerg Infect Dis 2007;13:1777-80.

11. American Academy of Pediatrics, Committee on Infectious Diseases. Red book: report of the Committee on Infectious Diseases. Elk Grove Village, IL: American Academy of Pediatrics; 1994:v.

12. World Health Organization. Drug-resistant salmonella. Available from: http://www.who.int/mediacentre/ factsheets/fs139/en/. Accessed January 17, 2011.

13. De Sarro A, De Sarro G. Adverse reactions to fluoroquinolones. An overview on mechanistic aspects. Curr Med Chem 2001;8:371-84.

14. Centers for Disease Control and Prevention, $\mathrm{Na}$ tional Center for Infectious Diseases. Healthy pets health people. Salmonella infection (salmonellosis) and animals. Available from: http://www.cdc.gov/ healthypets/diseases/salmonellosis.htm. Accessed January 17, 2011. 